\title{
Mixed generalized/juxta-articular form of Dercum's disease
}

\author{
Eugeniusz J. Kucharz, Justyna Kramza, Przemysław Kotyla, Anna Kotulska
}

Department of Internal Medicine and Rheumatology, Medical University of Silesia, Katowice, Poland

\begin{abstract}
Dercum's disease is a rare disorder with subcutaneous formation of fatty tissue (lipomas) with symptoms of pain, fatigue, stiffness, weakness and in some cases arthritis. The rarity of this disease causes that it is not taken into consideration in the rheumatological differential diagnosis, so this short report draws attention to this rare disease.
\end{abstract}

Key words: Dercum's disease, multiple fatty masses.

Dercum's disease is a rare condition characterized by painful fatty lumps located within the subcutaneous tissue. The disease is much more common in women and is associated with increased body mass and emotional disturbances. Dercum's disease appears in nodular, generalized and juxta-articular forms [1]. In this short report, we report a patient with mixed generalized/juxta-articular form.

A woman, a 40-year-old cosmetician and waitress, was seen in our department due to painful nodules widespread in the adipose tissue. The disease had begun one year earlier with pain in the left subcostal region. She discovered palpable small lumps. One of them was removed and pathological evaluation revealed that it had the structure of a lipoma. There were no systemic symptoms or concomitant disorders. The patient was overweight. She was not suffering from any other disease, either currently or in the past. The patient's menstruation had stopped spontaneously at a relatively young age (she was 39), a few weeks before the first appearance of the cutaneous symptoms. Her father and his relatives had suffered from diabetes.

Physical examination revealed very small lumps located within the adipose tissue in the upper part of the abdomen, on the upper extremities, and one large soft mass (about $1.5 \mathrm{~cm}$ ) close to the knees. The skin was unchanged over the lipomas. The lump near the knee had no effect on joint movement. Palpation of the affected skin was painful and spontaneous pain occurred as well. Laboratory data were normal but serum glucose level was near the upper range of the normal value, and the total cholesterol level was increased.

On the basis of these findings, we diagnosed Dercum's disease in mixed generalized/juxta-articular form. She fulfilled so-called cardinal symptoms (multiple fatty masses, overweight) and minor symptoms (weakness, anxiety, depression). Differential diagnosis excluded fibromyalgia, benign symmetric lipomatosis, lipoedema, adenolipomatosis, and panniculitis.

The patient responded well to medication with paracetamol administered in low doses. We recommended continuation of this treatment as well as monitoring for diabetes or glucose intolerance. Metabolic disturbances are common in patients with Dercum's disease [2].

We have reported this case due to the potential to underdiagnose or easily mistake Dercum's disease with other disorders as well as the unusual form of the disease presentation.

The authors declare no conflict of interest.

\section{References}

1. Hausson E, Sevnsson H, Brorson H. Review of Dercum's disease and proposal of diagnostic criteria, diagnostic methods, classification and management. Orphanet J Rare Dis 2012; 7: 23-38.

2. Szypuła I, Kotulska A, Szopa M, et al. Adiposis dolorosa with hypercholesterotemia and premature severe generalized atherosclerosis. Wiad Lek 2009; 62: 64-65.

\section{Address for correspondence:}

Eugeniusz J. Kucharz, Department of Internal Medicine and Rheumatology, Medical University of Silesia, Ziołowa 45/47, 40-635 Katowice,

Poland, e-mail: ejkucharz@poczta.onet.pl

Submitted: 27.07.2016; Accepted: 12.08.2016 